\title{
Approximate Solution Method of the Seventh Order KdV Equations by Decomposition Method
}

\author{
Nawal Abdullah Alzaid1, Bashayr Ali Alrayiqi2 \\ ${ }^{1}$ Department of Mathematics, Faculty of Science, University of Jeddah, Jeddah, KSA \\ ${ }^{2}$ Department of Mathematics, College of Science and Arts, King Abdulaziz University, Rabigh, KSA \\ Email: b.a.f.r2012@hotmail.com
}

How to cite this paper: Alzaid, N.A. and Alrayiqi, B.A. (2019) Approximate Solution Method of the Seventh Order KdV Equations by Decomposition Method. Journal of Applied Mathematics and Physics, 7, 2148-2155.

https://doi.org/10.4236/jamp.2019.79147

Received: June 14, 2019

Accepted: September 26, 2019

Published: September 29, 2019

Copyright () 2019 by author(s) and Scientific Research Publishing Inc. This work is licensed under the Creative Commons Attribution International License (CC BY 4.0).

http://creativecommons.org/licenses/by/4.0/

\begin{abstract}
In this paper, Adomian decomposition method (ADM) is implemented to approximate the solution of the Korteweg-de Vries (KdV) equations of seventh order, which are Kaup-Kuperschmidt equation and seventh order Kawahara equation. The results obtained by the $\mathrm{ADM}$ are compared with the exact solutions. It is found that the ADM is very efficient and convenient and can be applied to a large class of problems. The conservation properties of solution are examined by calculating the first three invariants.
\end{abstract}

\section{Keywords}

Adomian Decomposition Method, Kaup-Kuperschmidt Seventh-Order KdV Equation, Seventh-Order Kawahara Equation, Conservation Laws

\section{Introduction}

The general seventh-order KdV equation (gsKdV) reads

$$
\begin{aligned}
& u_{t}+a u^{3} u_{x}+b u_{x}^{3}+c u u_{x} u_{x x}+d u^{2} u_{x x x}+e u_{x x} u_{x x x} \\
& +f u_{x} u_{x x x x}+g u u_{x x x x x}+u_{x x x x x x}=0,
\end{aligned}
$$

where $a, b, c, d, e, f$ and $g$ are nonzero parameters. One of the well-known particular cases of Equation (1) is called seventh order Kaup Kuperschmidt equation (KK) [1] which can be shown in the form

$$
\begin{aligned}
& u_{t}+2016 u^{3} u_{x}+630 u_{x}^{3}+2268 u u_{x} u_{x x}+504 u^{2} u_{x x x}+252 u_{x x} u_{x x x} \\
& +147 u_{x} u_{x x x x}+42 u u_{x x x x x}+u_{x x x x x x x}=0,
\end{aligned}
$$

Another form of the seventh-order $\mathrm{KdV}$ equation is called seventh order Kawahara equation [2] which can be shown in the form 


$$
u_{t}+6 u u_{x}+u_{x x x}-u_{x x x x x}+\alpha u_{x x x x x x}=0,
$$

where $\alpha$ is a nonzero constant. These equations were introduced initially by Pomeau et al. [3] for discussing the structural stability of KdV equation under a singular perturbation. These equations play an important role in mathematical physics, engineering and applied sciences for investigating travelling solitary wave solutions.

The Adomian decomposition method (ADM) was first proposed by George Adomian in the 1980's [4] [5] [6] [7]. This technique has been shown to solve effectively, easily, and accurately a large class of linear and nonlinear, ordinary or partial, deterministic or stochastic differential equations with approximates which converge rapidly to accurate solutions. This method is well-suited to physical problems since it makes the unnecessary linearization, perturbation problem being solved, sometimes seriously. Conservation laws (CLaws) are of basic importance in the study of evolution equations because they provide physical, conserved quantities for all solutions $u(x, t)$, and they can be used to check the accuracy of numerical solution methods [8] [9] [10] [11] [12]. The paper is arranged in the following manner: in Section 2, we present the ADM; Section 3 presents the CLaws for (KK) and Kawahara seventh-order KdV equations [13] [14]; in Section 4, the ADM is implemented to some problems in addition to studying the properties of CLaws; finally, a brief conclusion is given in Section 5 .

\section{The Method of Solution}

Consider the (gsKdV) equation in an operator form

$$
\begin{aligned}
& L_{t}(u)+a(K u)+b(M u)+c(N u)+d(P u)+e(Q u) \\
& +f(R u)+g(V u)+L_{7 x}(u)=0,
\end{aligned}
$$

where the notations $K u=u^{3} u_{x}, M u=u_{x}^{3}, N u=u u_{x} u_{x x}, P u=u^{2} u_{x x x}$, $Q u=u_{x x} u_{x x x}, R u=u_{x} u_{x x x x}$ and $V u=u u_{x x x x x}$ symbolize the nonlinear terms, respectively. Also, the notation $L_{t}=\frac{\partial}{\partial t}$ and $L_{7 x}=\frac{\partial^{7}}{\partial x^{7}}$ symbolize the linear differential operators. Assuming $L_{t}^{-1}$ the inverse of operator of $L_{t}$ exists and conveniently by

$$
L_{t}^{-1}=\int_{0}^{t}(.) \mathrm{d} t
$$

Thus, applying the inverse operator $L_{t}^{-1}$ to (4) yields

$$
\begin{aligned}
u(x, t)= & h(x)-a L_{t}^{-1}(K u)-b L_{t}^{-1}(M u)-c L_{t}^{-1}(N u)-d L_{t}^{-1}(P u) \\
& -e L_{t}^{-1}(Q u)-f L_{t}^{-1}(R u)-g L_{t}^{-1}(V u)-L_{t}^{-1}\left(L_{7 x} u\right) .
\end{aligned}
$$

The standard ADM [15] defines the solution $u(x, t)$ by the decomposition series

$$
u(x, t)=\sum_{n=0}^{\infty} u_{n}(x, t),
$$

with $u_{0}$ identified as $u(x, 0)$. The nonlinear terms $K u, M u, N u, P u, Q u, R u$ 
and $V u$ can be decomposed into infinite series of polynomial given by

$$
\begin{aligned}
& K u=u^{2} u_{x}=\sum_{n=0}^{\infty} A_{n}, \\
& M u=u_{x} u_{x x}=\sum_{n=0}^{\infty} B_{n}, \\
& N u=u u_{x x x}=\sum_{n=0}^{\infty} C_{n}, \\
& P u=u u_{x x x}=\sum_{n=0}^{\infty} D_{n}, \\
& Q u=u u_{x x x}=\sum_{n=0}^{\infty} E_{n}, \\
& R u=u u_{x x x}=\sum_{n=0}^{\infty} F_{n}, \\
& V u=u u_{x x x}=\sum_{n=0}^{\infty} G_{n},
\end{aligned}
$$

where $A_{n}, B_{n}, B_{n}, D_{n}, E_{n}, F_{n}$ and $G_{n}$ are the so-called Adomian polynomials of $u_{0}, u_{1}, \cdots, u_{n}$ defined by equation

$$
P_{n}=\frac{1}{n !} \frac{\mathrm{d}^{n}}{\mathrm{~d} \lambda^{n}}\left[N\left(\sum_{i=0}^{\infty} \lambda^{i} u_{i}(x, t)\right)\right]_{\lambda=0}, n \geq 0 .
$$

The components $u_{n}(x, t)$ can be determined sequentially by the standard recursion scheme as:

$$
\left\{\begin{aligned}
& u_{0}(x, t)=h(x), \\
& u_{n+1}=-a L_{t}^{-1}\left(A_{n}\right)-b L_{t}^{-1}\left(B_{n}\right)-c L_{t}^{-1}\left(C_{n}\right)-d L_{t}^{-1}\left(D_{n}\right)-e L_{t}^{-1}\left(E_{n}\right) \\
&-f L_{t}^{-1}\left(F_{n}\right)-g L_{t}^{-1}\left(G_{n}\right)-L_{t}^{-1}\left(L_{7 x} u_{n}\right), n \geq 0 .
\end{aligned}\right.
$$

\section{Conservation Laws}

The conservation properties of the solution are examined by calculating the Claws.

1) For KK equation Equation (2), the conservative quantities $I_{i}(i=1,2,3)$ can be written as

$$
\begin{aligned}
& I_{1}=\int_{-\infty}^{\infty} u \mathrm{~d} x, \\
& I_{2}=\int_{-\infty}^{\infty}\left(u^{3}-\frac{1}{8} u_{x}^{2}\right) \mathrm{d} x, \\
& I_{3}=\int_{-\infty}^{\infty}\left(u^{4}-\frac{3}{4} u u_{x}^{2}+\frac{1}{48} u_{x x}^{2}\right) \mathrm{d} x,
\end{aligned}
$$

2) For seventh-order Kawahara equation Equation (3), the conservative quantities $I_{i}(i=1,2,3)$ can be written as

$$
\begin{aligned}
& I_{1}=\int_{-\infty}^{\infty} u \mathrm{~d} x, \\
& I_{2}=\int_{-\infty}^{\infty} u^{2} \mathrm{~d} x, \\
& I_{3}=\int_{-\infty}^{\infty}\left(-u^{3}+\frac{1}{2}\left(u_{x}\right)^{2}-\frac{1}{2}\left(u_{x x}\right)^{2}+\frac{1}{2} \alpha\left(u_{x x x}\right)^{2}\right) \mathrm{d} x,
\end{aligned}
$$


Since the conservation constants are expected to remain constant during the run of the algorithm to have accurate numerical scheme, conservation constants will be monitored. As various problems of science were modeled by non linear partial differential equations and since therefore the seventh order KdV equation is of high importance, the following examples have been considered.

\section{Numerical Examples}

Example 1. Consider the seventh-order (KK) equation Equation (2) with initial condition

$$
u(x, 0)=\frac{1}{3} k^{2}-\frac{1}{2} k^{2} \tanh ^{2}(k x),
$$

By ADM the recursive relations are

$$
\left\{\begin{aligned}
u_{0}= & \frac{1}{3} k^{2}-\frac{1}{2} k^{2} \tanh ^{2}(k x) \\
u_{n+1}= & -2016 L_{t}^{-1}\left(A_{n}\right)-630 L_{t}^{-1}\left(B_{n}\right)-2268 L_{t}^{-1}\left(C_{n}\right)-504 L_{t}^{-1}\left(D_{n}\right) \\
& \quad-252 L_{t}^{-1}\left(E_{n}\right)-147 L_{t}^{-1}\left(F_{n}\right)-42 L_{t}^{-1}\left(G_{n}\right)-L_{t}^{-1}\left(L_{7 x} u_{n}\right), n \geq 0 .
\end{aligned}\right.
$$

The first few components are thus determined as follows:

$$
\left\{\begin{array}{l}
u_{0}=\frac{1}{3} k^{2}-\frac{1}{2} k^{2} \tanh ^{2}(k x), \\
u_{1}=\frac{-4 k^{9} t \sinh (k x)}{3 \cosh ^{3}(k x)} \\
u_{2}=\frac{8 k^{16} t^{2}\left(2 \cosh ^{2}(k x)-3\right)}{9 \cosh ^{4}(k x)},
\end{array}\right.
$$

and so on. Consequently, the solution in a series form is given by

$$
u(x, t)=u_{0}+u_{1}+u_{2}+\cdots
$$

and in a closed form $u(x, t)=\frac{1}{3} k^{2}-\frac{1}{2} k^{2} \tanh ^{2}\left(k\left(x+\frac{4}{3} k^{6} t\right)\right)$.

The results produced by the proposed method with only few components ( $\mathrm{n}=$ 5) are compared with the exact solution and listed in Table 1, also the Claws for the seventh-order (KK) equation are given in Table 2. The profile of the solitary wave at $t=0.3$ is displayed in Figure 1.

Table 1. Comparison between exact solution $u(x, t)$ and approximate solution using ADM where $k=0.1$.

\begin{tabular}{cccc}
\hline $\boldsymbol{X}$ & Exact & ADM & Absolute Error \\
\hline 0.10 & 0.00333283 & 0.00333283 & $9.98865803 \mathrm{e}^{-19}$ \\
0.20 & 0.00333133 & 0.00333133 & $1.99693372 \mathrm{e}^{-18}$ \\
0.30 & 0.00332884 & 0.00332884 & $2.99340524 \mathrm{e}^{-18}$ \\
0.40 & 0.00332534 & 0.00332534 & $3.98748557 \mathrm{e}^{-18}$ \\
0.50 & 0.00332085 & 0.00332085 & $4.97838399 \mathrm{e}^{-18}$ \\
\hline
\end{tabular}


Table 2. Computed quantities $I_{1}, I_{2}, I_{3}$ for the seventh-order KK equation by ADM.

\begin{tabular}{|c|c|c|c|c|c|c|c|c|c|}
\hline & \multicolumn{3}{|c|}{$I_{1}$} & \multicolumn{3}{|c|}{$I_{2}$} & \multicolumn{3}{|c|}{$I_{3}$} \\
\hline$t / x$ & 0.1 & 0.3 & 0.5 & 0.1 & 0.3 & 0.5 & 0.1 & 0.3 & 0.5 \\
\hline 0.1 & $3.333 \mathrm{e}^{-4}$ & $9.996 \mathrm{e}^{-4}$ & $1.665 \mathrm{e}^{-3}$ & $3.703 \mathrm{e}^{-9}$ & $1.108 \mathrm{e}^{-8}$ & $1.840 \mathrm{e}^{-8}$ & $3.316 \mathrm{e}^{-11}$ & $9.910 \mathrm{e}^{-11}$ & $1.639 \mathrm{e}^{-10}$ \\
\hline 0.2 & $3.333 \mathrm{e}^{-4}$ & $9.996 \mathrm{e}^{-4}$ & $1.665 \mathrm{e}^{-3}$ & $3.703 \mathrm{e}^{-9}$ & $1.108 \mathrm{e}^{-8}$ & $1.840 \mathrm{e}^{-8}$ & $3.316 \mathrm{e}^{-11}$ & $9.910 \mathrm{e}^{-11}$ & $1.639 \mathrm{e}^{-10}$ \\
\hline 0.3 & $3.333 \mathrm{e}^{-4}$ & $9.996 \mathrm{e}^{-4}$ & $1.665 \mathrm{e}^{-3}$ & $3.703 \mathrm{e}^{-9}$ & $1.108 \mathrm{e}^{-8}$ & $1.840 \mathrm{e}^{-8}$ & $3.316 \mathrm{e}^{-11}$ & $9.910 \mathrm{e}^{-11}$ & $1.639 \mathrm{e}^{-10}$ \\
\hline 0.4 & $3.333 \mathrm{e}^{-4}$ & $9.996 \mathrm{e}^{-4}$ & $1.665 \mathrm{e}^{-3}$ & $3.703 \mathrm{e}^{-9}$ & $1.108 \mathrm{e}^{-8}$ & $1.840 \mathrm{e}^{-8}$ & $3.316 \mathrm{e}^{-11}$ & $9.910 \mathrm{e}^{-11}$ & $1.639 \mathrm{e}^{-10}$ \\
\hline 0.5 & $3.333 \mathrm{e}^{-4}$ & $9.996 \mathrm{e}^{-4}$ & $1.665 \mathrm{e}^{-3}$ & $3.703 \mathrm{e}^{-9}$ & $1.108 \mathrm{e}^{-8}$ & $1.840 \mathrm{e}^{-8}$ & $3.316 \mathrm{e}^{-11}$ & $9.910 \mathrm{e}^{-11}$ & $1.639 \mathrm{e}^{-10}$ \\
\hline
\end{tabular}

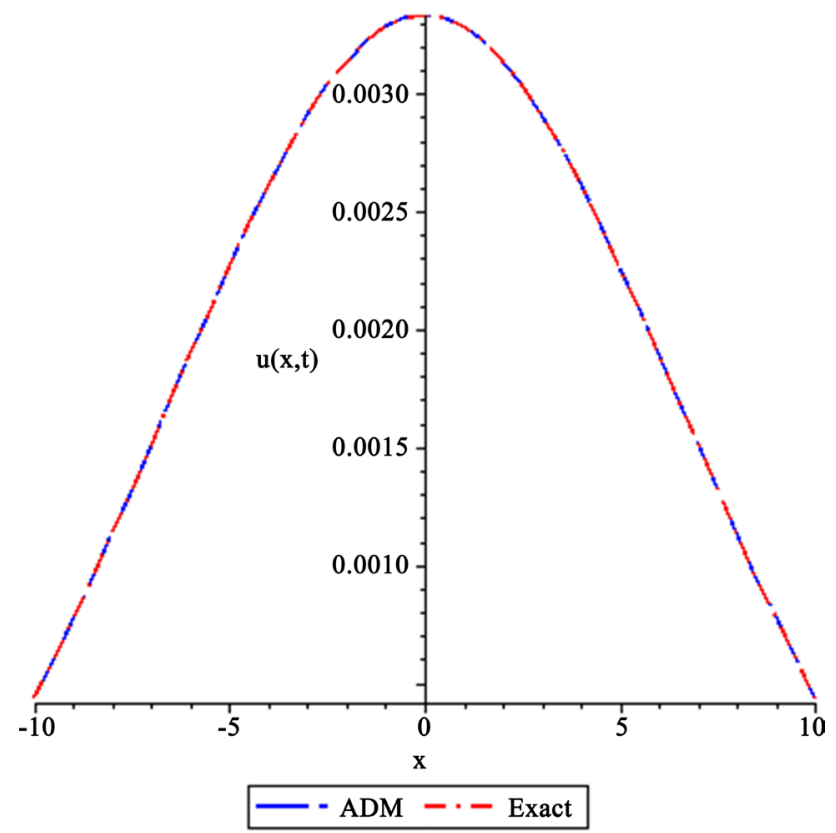

Figure 1. Comparison between exact solution $u(x, t)$ and approximate solution using ADM.

Example 2. Consider the seventh-order Kawahara equation Equation (3) with initial condition

$$
u(x, 0)=\operatorname{\omega sech}^{6}(k x),
$$

By $\mathrm{ADM}$ the recursive relations are

$$
\left\{\begin{array}{l}
u_{0}=\omega \operatorname{sech}^{6}(k x), \\
u_{n+1}=-6 L_{t}^{-1}\left(A_{n}\right)-L_{t}^{-1}\left(L_{3 x} u_{n}\right)+L_{t}^{-1}\left(L_{5 x} u_{n}\right)-\alpha L_{t}^{-1}\left(L_{7 x} u_{n}\right), n \geq 0 .
\end{array}\right.
$$

The first few components are thus determined as follows:

$$
\left\{\begin{aligned}
u_{0}= & \omega \operatorname{sech}^{6}(k x), \\
u_{1}= & \frac{1}{\cosh ^{13}(k x)}\left(1 2 t k \omega \operatorname { s i n h } ( k x ) \left(23328 \alpha k^{6} \cosh ^{6}(k x)\right.\right. \\
& \left.\left.-215488 \alpha k^{6} \cosh ^{4}(k x)-648 k^{4} \cosh ^{6}(k x)+\cdots\right)\right), \\
u_{2}= & \frac{1}{\cosh ^{20}(k x)}\left(1 2 t ^ { 2 } k ^ { 2 } \omega \left(108783285811200 \alpha^{2} k^{12} \cosh ^{6}(k x)\right.\right. \\
& -175649727052800 \alpha^{2} k^{12} \cosh ^{4}(k x) \\
& \left.\left.+138322888704000 \alpha^{2} k^{12} \cosh ^{2}(k x)+\cdots\right)\right),
\end{aligned}\right.
$$


and so on. Consequently, the solution in a series form is given by

$$
u(x, t)=u_{0}+u_{1}+u_{2}+\cdots
$$

and in a closed form $u(x, t)=\omega_{\operatorname{sech}^{6}}\left(k\left(x-x_{0} t\right)\right)$.

The results produced by the proposed method with only few components $(\mathrm{n}=$ 5) are compared with the exact solution and listed in Table 3, also the Claws for the seventh-order Kawahara equation are given in Table 4. The profile of the solitary wave at $t=0.3$ is displayed in Figure 2 .

Table 3. Comparison between exact solution $u(x, t)$ and approximate solution using ADM where $\alpha=\frac{769}{2500}, \omega=\frac{86625}{591361}, k=\frac{5}{\sqrt{1538}}$ and $x_{0}=\frac{180000}{591361}$.

\begin{tabular}{cccc}
\hline$x$ & Exact & ADM & Absolute Error \\
\hline 0.10 & 0.14648359 & 0.14648359 & $1.24475423 \mathrm{e}^{-11}$ \\
0.20 & 0.14639977 & 0.14639977 & $2.70176889 \mathrm{e}^{-11}$ \\
0.30 & 0.14617341 & 0.14617341 & $4.13404041 \mathrm{e}^{-11}$ \\
0.40 & 0.14580523 & 0.14580523 & $5.52856164 \mathrm{e}^{-11}$ \\
0.50 & 0.14529642 & 0.14529642 & $6.87280812 \mathrm{e}^{-11}$ \\
\hline
\end{tabular}

Table 4. Computed quantities $I_{1}, I_{2}, I_{3}$ for the seventh-order Kawahara equation by ADM.

\begin{tabular}{cccccccccc}
\hline \multicolumn{7}{c}{$I_{1}$} & \multicolumn{7}{c}{$I_{2}$} \\
\hline$t / X$ & 0.1 & 0.3 & 0.5 & 0.1 & 0.3 & 0.5 & 0.1 & 0.3 & 0.5 \\
\hline 0.1 & $1.465 \mathrm{e}^{-2}$ & $4.390 \mathrm{e}^{-2}$ & $7.300 \mathrm{e}^{-2}$ & $2.146 \mathrm{e}^{-3}$ & $6.424 \mathrm{e}^{-3}$ & $1.066 \mathrm{e}^{-2}$ & $-3.245 \mathrm{e}^{-4}$ & $-9.697 \mathrm{e}^{-4}$ & $-1.602 \mathrm{e}^{-3}$ \\
0.2 & $1.465 \mathrm{e}^{-2}$ & $4.391 \mathrm{e}^{-2}$ & $7.304 \mathrm{e}^{-2}$ & $2.146 \mathrm{e}^{-3}$ & $6.428 \mathrm{e}^{-3}$ & $1.067 \mathrm{e}^{-2}$ & $-3.245 \mathrm{e}^{-4}$ & $-9.708 \mathrm{e}^{-4}$ & $-1.606 \mathrm{e}^{-3}$ \\
0.3 & $1.465 \mathrm{e}^{-2}$ & $4.392 \mathrm{e}^{-2}$ & $7.308 \mathrm{e}^{-2}$ & $2.145 \mathrm{e}^{-3}$ & $6.430 \mathrm{e}^{-3}$ & $1.068 \mathrm{e}^{-2}$ & $-3.244 \mathrm{e}^{-4}$ & $-9.716 \mathrm{e}^{-4}$ & $-1.609 \mathrm{e}^{-3}$ \\
0.4 & $1.464 \mathrm{e}^{-2}$ & $4.393 \mathrm{e}^{-2}$ & $7.311 \mathrm{e}^{-2}$ & $2.145 \mathrm{e}^{-3}$ & $6.432 \mathrm{e}^{-3}$ & $1.069 \mathrm{e}^{-2}$ & $-3.242 \mathrm{e}^{-4}$ & $-9.721 \mathrm{e}^{-4}$ & $-1.612 \mathrm{e}^{-3}$ \\
0.5 & $1.464 \mathrm{e}^{-2}$ & $4.393 \mathrm{e}^{-2}$ & $7.313 \mathrm{e}^{-2}$ & $2.143 \mathrm{e}^{-3}$ & $6.433 \mathrm{e}^{-3}$ & $1.070 \mathrm{e}^{-2}$ & $-3.239 \mathrm{e}^{-4}$ & $-9.722 \mathrm{e}^{-4}$ & $-1.614 \mathrm{e}^{-3}$
\end{tabular}

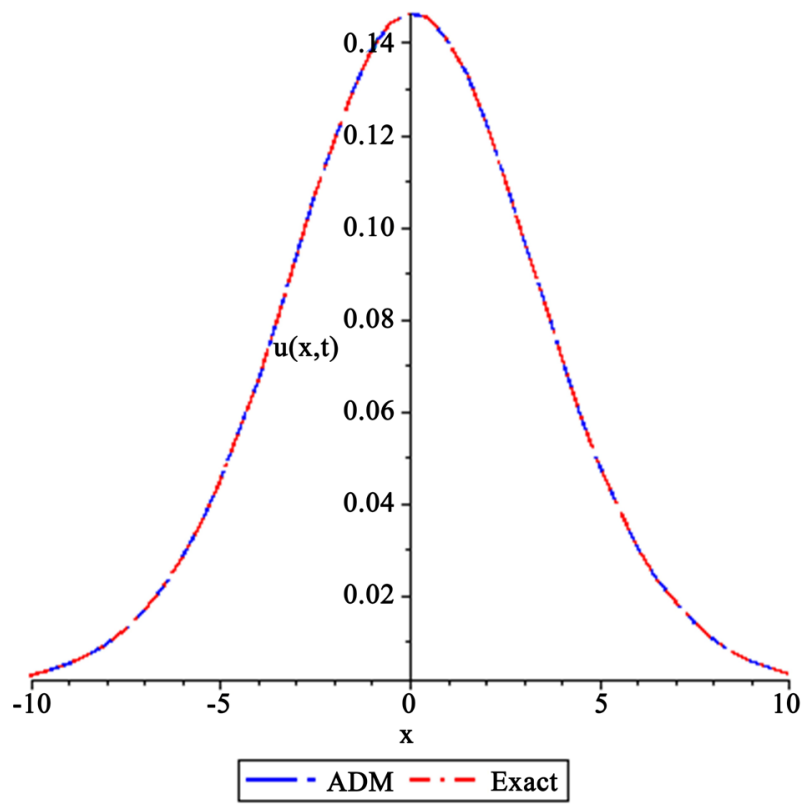

Figure 2. Comparison between exact solution $u(x, t)$ and approximate solution using ADM. 


\section{Conclusion}

In this paper, the $\mathrm{ADM}$ was used to solving seventh order KdV equations with initial conditions. We have found out that this method is applicable and efficient technique. All the numerical results obtained by using ADM show very good agreement with the exact solutions for a few terms. The conservation laws are used to assess the accuracy and the efficiency of the method. We have noticed that the method accomplished the aim of preserving conserved quantities, as we saw all invariants were almost constant.

\section{Conflicts of Interest}

The authors declare no conflicts of interest regarding the publication of this paper.

\section{References}

[1] Fan, E. and Hona, Y.C. (2002) Generalized Tanh Method Extended to Special Types of Nonlinear Equations. Zeitschrift fur Naturforschung A, 57, 692-700. https://doi.org/10.1515/zna-2002-0809

[2] Wazwaz, A.M. (2011) Soliton Solutions for Seventh-Order Kawahara Equation with Time-Dependent Coefficients. Modern Physics Letters B, 25, 643-648. https://doi.org/10.1142/S0217984911026012

[3] Pomeau, Y., Ramani, A. and Grammaticos, B. (1988) Structural Stability of the Korteweg-de Vries Solitons under a Singular Perturbation. Physica D: Nonlinear Phenomena, 31, 127-134. https://doi.org/10.1016/0167-2789(88)90018-8

[4] Adomian, G. (1988) A Review of the Decomposition Method in Applied Mathematics. Journal of Mathematical Analysis and Applications, 135, 501-544. https://doi.org/10.1016/0022-247X(88)90170-9

[5] Adomian, G. (1991) Solving Frontier Problems Modelled by Nonlinear Partial Differential Equations. Computers and Mathematics with Applications, 22, 91-94. https://doi.org/10.1016/0898-1221(91)90017-X

[6] Adomian, G. (1986) A New Approach to the Heat Equation: An Application of the Decomposition Method. Journal of Mathematical Analysis and Applications, 113, 202-209. https://doi.org/10.1016/0022-247X(86)90344-6

[7] Adomian, G. (1983) Stochastic Systems. Academic Press, New York.

[8] Olver, P.J. (2012) Applications of Lie Groups to Differential Equations. Springer Science and Business Media, Berlin, 107.

[9] Bluman, G.W., Cheviakov, A.F. and Anco, S.C. (2010) Applications of Symmetry Methods to Partial Differential Equations. Springer, New York. https://doi.org/10.1007/978-0-387-68028-6

[10] Kashkari, B.S. and Bakodah, H.O. (2013) New Modification of Laplace Decomposition Method for Seventh-Order KdV Equation. AIP Conference Proceedings, 1558, 2435-2439. https://doi.org/10.1063/1.4826033

[11] Bakodah, H.O. and Banaja, M.A. (2013) The Method of Lines Solution of the Regularized Long-Wave Equation Using Runge-Kutta Time Discretization Method. Mathematical Problems in Engineering, 2013, Article ID: 804317.

https://doi.org/10.1155/2013/804317

[12] Banaja, M.A. and Bakodah, H.O. (2015) Runge-Kutta Integration of the Equal 
Width Wave Equation Using the Method of Lines. Mathematical Problems in Engineering, 2015, Article ID: 804317. https://doi.org/10.1155/2015/274579

[13] Yao, R.-X. and Li, Z.-B. (2004) Conservation Laws and New Exact Solutions for the Generalized Seventh Order KdV Equation. Chaos, Solitons and Fractals, 20, 259-266. https://doi.org/10.1016/S0960-0779(03)00373-4

[14] Yao, R.-X., Xu, G.-Q. and Li, Z.-B. (2004) Conservation Laws and Soliton Solutions for Generalized Seventh Order KdV Equation. Communications in Theoretical Physics, 41, 487-492. https://doi.org/10.1088/0253-6102/41/4/487

[15] Adomian, G. (1984) A New Approach to Nonlinear Partial Differential Equations. Journal of Mathematical Analysis and Applications, 102, 420-434.

https://doi.org/10.1016/0022-247X(84)90182-3 